\title{
Erratum
}

\section{Relativistic calculation of Stokes' parameters for intermediate energy electron impact excitation of cesium atoms}

\author{
V. Zeman, R.P. McEachran, A.D. Stauffer
}

Department of Physics and Astronomy, York University, Toronto, Ontario, Canada M3J 1 P3

Z. Phys. D 30, 145-148 (1994)

We have found a computational error in our RDW computer algorithm which has affected the results previously published. We therefore show the revised version of our results here.

Figures 1-3 show the various differential Stokes' parameters at an incident electron energy of $20 \mathrm{eV}$. The behaviour of these parameters differs substantially from the published results except for small scattering angles and near 180 degrees.

In Fig. 4 we show the integrated Stokes' parameter $\eta_{3}^{y}$ for the transition $(6 p){ }^{2} P_{3 / 2} \rightarrow(6 s){ }^{2} S_{1 / 2}$. This parameter is now seen to lie in between the experimental data of Eschen et a1. [6] and Chen and Gallagher [7]. In Fig. 5 we show the integrated parameters $\eta_{2}^{y} / P_{y}$ and $\eta_{1}^{y} / P_{y}$ for the same transition. $\eta_{2}^{y} / P_{y}$ is roughly the same shape as before but has decreased in value by approximately a factor of 10. Although our results are of the same order of magnitude as the experimental values of Eschen et al. [6], they differ in sign. Our results for $\eta_{1}^{y} / P_{y}$ are now extremely

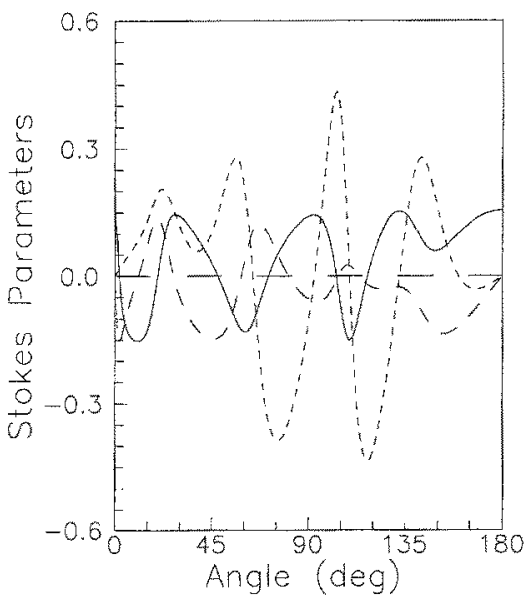

Fig. 1. Differential Stokes' parameters for the transition $(6 p)$ ${ }^{2} P_{3 / 2} \rightarrow(6 s){ }^{2} S_{1 / 2}$ in cesium after impact excitation by unpolarized electrons with an incident energy of $20 \mathrm{eV} . \ldots, \eta_{3}^{y} ;--,, \eta_{1}^{y} ; \ldots$ $\eta_{2}^{y}$

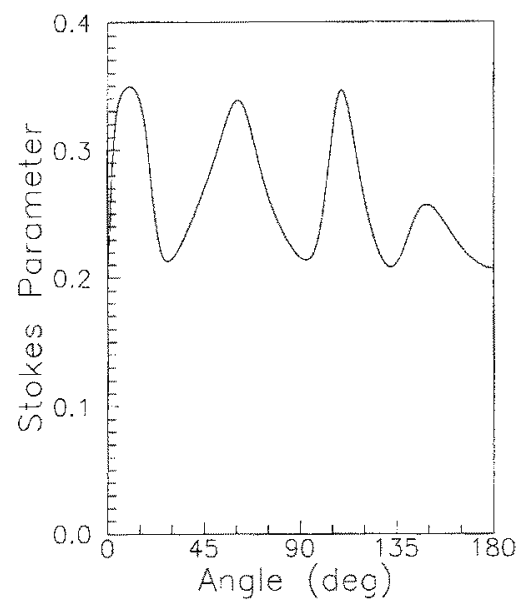

Fig. 2. As in Fig. 1 for $\eta_{2}^{2} / P_{\text {atom }}$ where $P_{\text {atom }}$ is the spin polarization of the atom in its initial state

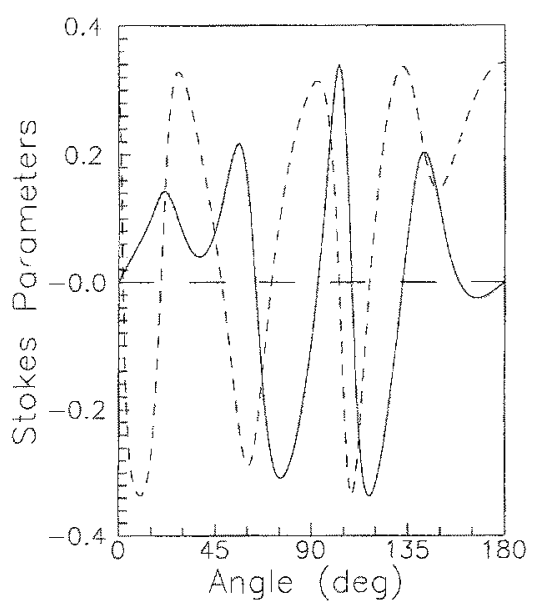

Fig. 3. Differential Stokes parameters for the transition (6p) ${ }^{2} P_{1 / 2} \rightarrow(6 s){ }^{2} S_{1 / 2}$ in cesium after impact excitation by unpolarized electrons with an incident energy of $20 \mathrm{eV}$. - - , $\eta_{2}^{y} ;---, \eta_{2}^{z} / P_{\text {atom }}$ 


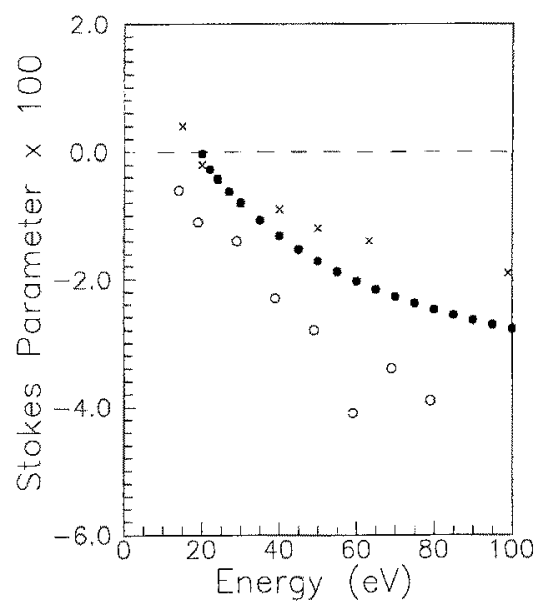

Fig. 4. Integrated Stokes' parameter $\eta_{3}^{y}$, multiplied by a factor of 100 , for the transition $(6 p){ }^{2} P_{3 / 2} \rightarrow(6 s)^{2} S_{3 / 2}$ in cesium after impact excitation by electrons polarized in the $y$-direction. Present data; $O$, experimental results of Eschen et al. [6]; $x$, experimental results of Chen and Gallagher [7]

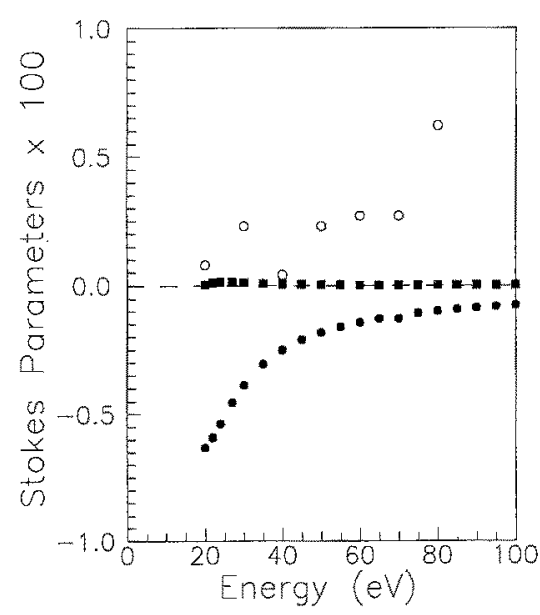

Fig. 5. Integrated Stokes' parameters, multiplied by a factor of 100 , for the transition $(6 p){ }^{2} P_{3 / 2} \rightarrow(6 s){ }^{2} S_{1 / 2}$ in cesium after impact excitation by electrons polarized in the $y$-direction. Present data for $\eta_{2}^{y} / P_{y} ; 0$, experimental results of Eschen et al. [6] for $\eta_{2}^{y} / P_{y} ; \mathbf{\square}$, present data for $\eta_{1}^{y} / P_{y}$. Here $P_{y}$ is the polarization of the incident electrons

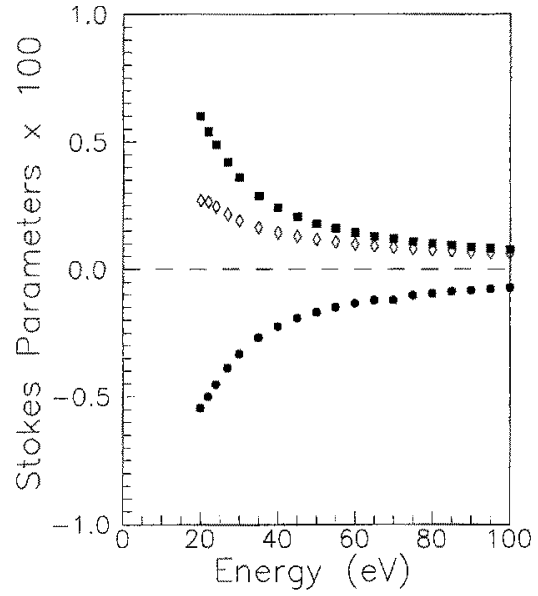

Fig. 6. Integrated Stokes' parameters, multiplied by a factor of 100 , in cesium after impact excitation by polarized electrons. $\eta_{2}^{2} / P_{z}$ for the transition $(6 p)^{2} P_{3 / 2} \rightarrow(6 s)^{2} S_{1 / 2} ; \mathbf{E}, \eta_{2}^{y} / P_{y}$ for the transition $(6 p)$ ${ }^{2} P_{1 / 2} \rightarrow(6 s)^{2} S_{1 / 2} ; 0, \eta_{2}^{z} / P_{z}$ for the transition $(6 p){ }^{2} P_{1 / 2} \rightarrow(6 s)^{2} S_{1 / 2}$. The electrons are polarized in the same direction as the orientation of the photon detector

close to zero in value. In Fig. 6 we show $\eta_{2}^{z} / P_{z}$ for the $(6 p)$ ${ }^{2} P_{3 / 2} \rightarrow(6 s){ }^{2} S_{1 / 2}$ transition as well as the two circular integrated Stokes' parameters for the $(6 p){ }^{2} P_{1 / 2} \rightarrow(6 s)$ ${ }^{2} S_{1 / 2}$ transition. The $\eta_{2}^{y} / P_{y}$ parameter for the $(6 p)$ ${ }^{2} P_{3 / 2} \rightarrow(6 s){ }^{2} S_{1 / 2}$ transition has not changed appreciably. For the $(6 p){ }^{2} P_{1 / 2} \rightarrow(6 s){ }^{2} S_{1 / 2}$ transition, $\eta_{2}^{z} / P_{z}$ has decreased by approximately $1 / 2$ while $\eta_{2}^{y} / P_{y}$ has changed in both magnitude and sign.

We also update our reference list here:

3. Zeman, V., McEachran, R.P., Stauffer, A.D.: J. Phys, B27, 3175 (1994)

Zeitschrift für Physik D is delivered to some institutions in the former Soviet Union through a program initiated and administered by the European Physical Society and supported by the publisher. This program is funded by INTAS, the international association for the promotion of cooperation with scientists from the independent States of the former Soviet Union. Members of INTAS are the European Union, Austria, Belgium, Denmark, Finland, France, Germany, Greece, Ireland, Italy, Luxembourg, The Netherlands, Portugal, Spain, United Kingdom and Switzerland. (INTAS, rue du Luxembourg 14A, 1040 Bruxelles, Belgique). 